\title{
Reflexiones y Propuestas en Torno al Dilema entre Campo Disciplinar y Quehacer Profesional: Prácticas, Políticas y Organización
}

\author{
Reflections and Proposals Regarding the Dilemma Between Disciplinary \\ field and Professional Work: Practices, Policies and Organization
}

\author{
Ramón Fuentes Fernández ${ }^{1,2}$; Carlos Del Valle Rojas³; \\ Claudia Riquelme Macalusso ${ }^{4}$; Marcos Calle Recabarren ${ }^{5}$
}

FUENTES, F. R.; DEL VALLE, R. C.; RIQUELME, M. C. \& CALLE, R. M. Reflexiones y propuestas en torno al dilema entre campo disciplinar y quehacer profesional: prácticas, políticas y organización. Int. J. Odontostomat., 14(4):581-585, 2020.

RESUMEN: El presente artículo reflexiona sobre cómo la sociedad del conocimiento actual entiende el concepto "gobernanza universitaria", respecto de su producción científica. De igual modo, planteamos que el quehacer científico de las universidades es dinámico, pues deben proponer adaptaciones según los momentos de cambios y transformaciones sociales que experimentan en su interior las casas de estudios superiores.

PALABRAS CLAVE: gobernanza, gestión, productividad científica.

\section{INTRODUCCIÓN}

El objetivo del presente trabajo es abordar las lógicas y dinámicas que se producen al interior de la Universidad, a partir de las tensiones entre campo disciplinar y quehacer profesional, especialmente en los procesos sociales de construcción de prácticas epistémicas o cognitivas Olivé (2019), y la sistematización de conocimientos o "ecología de saberes" De Sousa (2009) que son inherentes a los fines la universidad, en tanto orientan y articulan el desarrollo académico y profesional, dentro del marco organizacional de la universidad. Vale decir, la función principal de los miembros de la comunidad universitaria es producir saberes o conocimientos y transferirlos adecuadamente, ya sea, tanto en la formación profesional - título-, como en la formación disciplinar - licenciatura -, en cuyo caso la estructura organizacional debería potenciar ambas prácticas y evitar el estancamiento o debilitamiento de alguno de los ámbitos de desarrollo académico. En este sentido, las diferentes estructuras organizacionales constituyen un ámbito que debe propiciar, por un parte, el desarrollo teórico y práctico de políticas pertinentes de distribución —asignación o liberación de horas, tipos de contrato, y demás- y políticas de reconocimiento - sistemas de incentivos, carrera académica, categorización y otras-, considerando las diferencias existentes en las culturas disciplinares y profesionales. Por tanto, es importante repensar y revisar de forma crítica nuestros enunciados organizacionales, a saber, las certezas de la razón moderna, el progreso de la ciencia y el optimismo en las profesiones Mollis (2006) que han prevalecido en universidades de América Latina.

Por lo anterior, este artículo aborda en el primer apartado los modos de gestión del conocimiento, partiendo con la comprensión de la naturaleza actual de la actividad cognitiva y luego ubicando la reflexión en dos estrategias hoy en uso y sus implicancias posibles, a saber, las nuevas políticas de incentivo y contratación. Luego, las dos secciones siguientes abor-

\footnotetext{
${ }^{1}$ Centro de investigación en Ciencias Odontológicas (CICO), Facultad de Odontología, Universidad de La Frontera, Temuco, Chile.

2 Departamento de Odontología Integral Adultos, Facultad de Odontología, Universidad de La Frontera, Temuco, Chile.

${ }^{3}$ Doctorado en Comunicación, Universidad de La Frontera, Temuco, Chile.

${ }^{4}$ Facultad de Odontología, Universidad de La Frontera, Temuco, Chile.

${ }_{5}^{5}$ Departamento de Historia y Geografía, Facultad de Comunicación, Historia y Ciencias Sociales de la Universidad Católica de la Santísima Concepción, Concepción, Chile.
} 
FUENTES, F. R.; DEL VALLE, R. C.; RIQUELME, M. C. \& CALLE, R. M. Reflexiones y propuestas en torno al dilema entre campo disciplinar y quehacer profesional: prácticas, políticas y organización. Int. J. Odontostomat., 14(4):581-585, 2020.

dan los modos de producción del conocimiento, con énfasis en políticas de investigación y publicaciones indizadas, por sobre otras actividades académicas; y finalmente las transformaciones académico-culturales y exigencias éticas asociadas a la producción científica considerando las nuevas condiciones y reglas.

Gobernanza universitaria. La sociedad del conocimiento se ha orientado a tratar los fenómenos de interacciones, no tan solo de los actores públicos, sino que también de los actores privados, lo cual ha tenido una injerencia relevante en como las universidades visibilizan y amplían las temáticas de la sociedad donde se desenvuelve. En ese sentido, las universidades como organizaciones generadoras de conocimiento, tienen un rol integrador en el contexto donde se vinculan, ya sean en el ámbito político, social y productivo en los lugares donde se insertan, y por tanto, deben profesionalizar su gestión y fortalecer las funciones administrativas de forma sistemática en el último tiempo. Finalmente, las casas de estudios superiores han buscado equilibrar el cuerpo académico y administrativo universitario, a través de un modelo de gestión basado en la sustentabilidad económica y financiera, así como también, mejorar la calidad formativa y producción científica que promueve.

Es bien sabido, que las universidades en el mundo han producido profundas transformaciones a nivel global, nacional y regional en sus formas de gestionar y administrar el sistema de educación superior; pues al ser organizaciones que producen capital humano avanzado, han tenido que reflexionar y modificar sus modelos de gestión para responder a los nuevos desafíos y requerimientos que las comunidades locales demandan por más perfeccionamiento profesional y continuidad de estudios universitarios. Confirman lo anterior Fossatti y Danesi (2018) "estos cambios implican el desarrollo de modelos de gestión que atiendan a los desafíos de una nueva economía y sociedad con sustentabilidad" (p. 76). Colegimos, que actualmente las universidades no pueden solamente funcionar según las capacidades instaladas dentro de la comunidad universitaria, sino que deben trabajar colaborativamente con otras universidades en aras del desarrollo científico, tanto nacional como internacional, para y así lograr coherencia en los contextos donde se desenvuelve, y mejorar la gobernanza universitaria. Este último concepto, constituye un espacio de interacción interdependiente entre universidades, organizaciones civiles y las comunidades locales, y como plantea Schmal \& Cabrales (2018) es "un conjunto interrelacionado de elementos asociados a la autonomía universitaria, la organización y gestión económi- co-financiera, las estructuras académicas en que se distribuyen las funciones y las responsabilidades, la organización del quehacer académico, las relaciones institucionales e internacionales y su promoción en el exterior" (p. 828). Por tanto, la gobernanza universitaria, debería reflejar el modelo universitario de cada institución superior, y además un constructo que evidencie las necesidades internas del sistema académico. Finalmente, la gobernanza universitaria debe abrir espacios que aseguren la participación interna de la comunidad universitaria, para responder las complejidades de la realidad social local y también incidir e impactar en la sociedad del conocimiento (Rock \& Rojas, 2012; Arias Arbelaez \& Vargas, 2010; Alzate Zuluaga \& Romo Morales, 2014; Virgili Lillo et al., 2015; Brunner \& Pedraja-Rejas, 2017; Castillo-Cubillos, 2017; Ganga et al., 2017; Schmal \& Cabrales).

Las políticas para la gestión del conocimiento. Actualmente, uno de los principales desafíos de la universidad es definir políticas adecuadas para cumplir su principal propósito: producir conocimiento transferible, tanto de divulgación, como formativos y de intervención social y cultural. Siendo esta su misión principal una parte importante de su esfuerzo deberá estar en el tipo de infraestructura y en su modelo organizacional a fin de crear las condiciones mínimas, necesarias y adecuadas para constituirse en una entidad capaz de producir conocimiento autónomo, básico o aplicado. Los estudios de Bourdieu (2003), Blondeau et al. (2004), proponen que la gestión del conocimiento se produce en un escenario caracterizado, al menos por:

a) El desarrollo del "capitalismo cognitivo", en cuyo caso la producción de conocimiento se sitúa en un mercado, con lógicas de oferta y demanda (¿quién decide qué producir? ¿cuál es el rol de la universidad, en el proceso de transacción e intercambio de conocimiento? ¿actualmente la universidad puede seguir pensándose solo a sí misma? ¿cómo identificar, los roles de oferta y demanda? ¿dónde está hoy situada la oferta y demanda?; con racionalidades de acumulación y niveles de concentración (indexación y patentización del conocimiento, etc.).

b) Las complejas "relaciones entre campo académico y profesional" al interior de nuestras universidades, con procesos de búsqueda de espacios de desarrollo, definición de núcleos, centros y líneas de investigación, nuevas lógicas de adscripción a la universidad, diferentes estatus científico, diversas formas de legitimación del conocimiento, etc. En este sentido, por ejem- 
FUENTES, F. R.; DEL VALLE, R. C.; RIQUELME, M. C. \& CALLE, R. M. Reflexiones y propuestas en torno al dilema entre campo disciplinar y quehacer profesional: prácticas, políticas y organización. Int. J. Odontostomat., 14(4):581-585, 2020.

plo, facultades, departamentos, escuelas, y demás, se fundan a partir de una operación de clasificación, donde "la clasificación es una condición del conocimiento, no el conocimiento mismo, y el conocimiento vuelve a disolver la clasificación" Horkheimer \& Adorno (1998). Del mismo modo, debemos entender cualquier forma de organización y clasificación como instancias de regulación que junto con ser entidades de apoyo y gestión, constituyen unidades de regulación cognitiva, económica y política. Cognitiva, porque inevitablemente delimita los territorios mentales; Económica, ya que, distribuye la escasez de recursos, bajo una racionalidad competitiva y contractual o convenida; y política, pues suponen decisiones unilaterales, expresadas en agendas, tiempos, la configuración de grupos de gestión, en instancias supradisciplinarias.

Desde esta perspectiva del análisis, podemos observar una dicotomía, en ocasiones tensionada, entre dos acciones políticas de gestión. Ambas apuntan a aspectos diferentes del trabajo universitario, por cierto, de la vida en sentido general, pero su propósito es el mismo, razón por la cual debemos destinar mayores esfuerzos:

a) Las políticas de incentivo, en tanto de reconocimiento, orientadas a la atracción y retención de capital humano avanzado. La discusión aquí es, inevitablemente, ¿dónde están los incentivos?: básicamente estarán donde estén los recursos estatales. Tanto los fondos estructurales (matrículas) como variables (productividad). Las políticas en este sentido pueden generar transformaciones significativas, que van desde cambiar el lugar de lo estructural y de lo variable hasta una hipertrofia en el desarrollo. Donde esté el incentivo encontraremos las mayores energías del recurso humano, porque, todos tomamos como base lo estructural y fijo y de manera casi instintiva buscaremos las fuentes de recursos adicionales. Algunas preguntas fundamentales aquí son: ¿cuál/es de las actividades académicas constituyen la base de nuestra actividad? Si es la docencia, ¿es conveniente incentivar la docencia? ¿hasta qué punto? Por otra parte, ¿cómo incentivar de mejor manera la actividad productiva en investigación y publicaciones? ¿cómo planificar adecuadamente para que el incentivo a la productividad, por un lado, permita obtener mayores recursos y, por otro, dejar capacidades instaladas? Si todas las universidades operan con racionalidad en los incentivos, lo que tenemos es una gran competencia por la atracción, centrada en una relación estrictamente económica, en vez de propiciar relaciones sociales. En suma, el objetivo es lograr que la organización reconozca adecuadamente sus actividades y a quienes las realizan, asignando un valor explícito y objetivable.

b) Las políticas de contratación, en tanto de distribución, orientadas a la consolidación de un capital humano calificado. La problemática es ¿qué formas de relación contractual propiciar? ¿cómo afecta el tipo de relación contractual al trabajo académico y la productividad científica? ¿cuándo hablar de flexibilidad y precariedad? El objetivo es lograr que la organización perciba una lógica de distribución racional, eficiente y coherente de los recursos, asignando valor referencial objetivo a las diferentes actividades. En un sistema universitario que promueve incentivos, es fácil perder de vista la actividad principal y necesaria. Este es, de hecho, uno de los principales debates al interior: ¿cuál es el lugar de la docencia de pregrado? ¿qué es más relevante? ¿qué es lo básico y qué es lo complementario? El contexto referido tiende a generar tensiones donde no deberíamos tenerlas.

La relación entre investigación y publicaciones. Esta relación, que siempre ha sido considerada fundamental en la actividad universitaria, especialmente por su interacción con la docencia y la extensión, en tanto productos transferibles social y culturalmente, hoy experimentan un desarrollo hipertrofiado, dado que se perciben como actividades que, concentran la distribución y el reconocimiento. Por consiguiente, ha obligado a realizar acciones improvisadas y de cuestionable resultado, como compensar mediante incentivos la labor docente. Su principal problema es que nunca se logrará tal compensación, por una parte, hay que considerar tanto lo material, como lo simbólico, y por otra, el sistema de incentivos sobre la productividad cada vez profundiza la brecha, debido a los niveles obsesivos de distinción de la productividad, que al tipo de índice (WoS, Scopus o SciELO), suma otras variables como el factor de impacto, en un in crescendo económico y simbólico. En este sentido, algunas consideraciones claves son comprender: ¿cómo las actuales condiciones del trabajo académico, implican transformaciones en la cultura organizacional de las universidades?, ¿qué sucede cuando el valor está en indicadores externos al trabajo intelectual, y cuando se imponen los procedimientos productivos y las exigencias de producción?.

Las condiciones de productividad científica. Es importante considerar la productividad científica, como la investigación y las publicaciones, en tanto prácticas epistémicas y disciplinarias que implican una institucionalidad específica —universidad, facultades, 
FUENTES, F. R.; DEL VALLE, R. C.; RIQUELME, M. C. \& CALLE, R. M. Reflexiones y propuestas en torno al dilema entre campo disciplinar y quehacer profesional: prácticas, políticas y organización. Int. J. Odontostomat., 14(4):581-585, 2020.

núcleos, centros y otros-, cierto estatus científico (tipos de reconocimiento científico, factor de impacto, etc.), disciplina versus profesión, culturas académicas (internacionalización, polivalencia, trabajo en red).

Esta última sección no puede dejar de ser también un breve ensayo sobre la ética en el trabajo intelectual y para evitar caer en posturas doctrinarias, más bien se planteará algunos itinerarios posibles para la discusión actual:

a) Cómo las transformaciones de la cultura académica inciden en el conocimiento, su producción y difusión. Aquí es necesario algunas precisiones, tales como la necesaria distinción entre el volumen y capacidad de producción de conocimiento, según la escala de la organización, frente a los desafíos de seguridad y confiabilidad. Más no es mejor.

b) Cómo la producción científica, nos exige abordar complejidades crecientes en nuestras universidades, como la distinción entre producción y procedimientos productivos, tiempo destinado a la producción y exigencias del trabajo académico, entre otras.

c) Cómo comprender los nuevos escenarios del trabajo académico, considerando aspectos como las exigencias de la propiedad intelectual, la necesidad de crear conciencia sobre nuevas formas de producción intelectual y, especialmente, la comprensión del rol y lugar del intelectual, y si seguimos pensando en el académico universitario como intelectual.

d) Cómo las formas de producción del conocimiento implican nuevos desafíos, como el rol de las tecnologías que construyen un archivo informatizado de nuestra memoria cognitiva, por ejemplo, cuando dicha memoria está en otro lugar o en ninguna parte.

e) Cómo el ethos académico o cultura académica se transforma en una tecno-ética, especialmente cuando la tecnología es un medio de producción de conocimiento y no solo de difusión. No solo se trata de bases de datos de revistas e índices, sino también de softwares.

f) Cómo la producción de conocimiento implica alcances éticos que exigen lógicas de autorregulación, donde la normativa (desde la cual acostumbrábamos a regular) se reduce a un tecnicismo (posibilidades de burlar la normativa), el trabajo intelectual implica ciertas pericias, el contexto de industrialización del conocimiento implica abusos, como el plagio; y donde tam- bién se ponen en juego el engaño regulado (lo que exigen las revistas), el autoengaño (¿autoplagio?) y el surgimiento de nuevas formas de engaño.

Algunas consideraciones finales. El propósito de este trabajo ha sido situar la discusión sobre la gobernanza universitaria, y no en factores puramente externos, como las condiciones económicas del financiamiento estatal, la competitividad público/privada, o las condiciones electorales; sino más bien en aspectos de decisión organizacional interna, esto es, cómo la universidad interpreta las condiciones externas y en base a las mismas establece estrategias.

Todo lo anterior desde un dilema transversal a las prácticas y condiciones señaladas: es necesaria la distinción entre campo disciplinar y quehacer profesional que sustentan todas las actuaciones en la organización universitaria. En este sentido, parte importante de cualquier estrategia implica comprender adecuadamente cuando se trata de operaciones en el campo disciplinario y cuando son acciones en el ámbito profesional. La adecuada convivencia de ambas es una conditio sine qua non de la existencia de la universidad.

FUENTES, F. R.; DEL VALLE, R. C.; RIQUELME, M. C. \& CALLE, R. M. Reflections and proposals regarding the dilemma between disciplinary field and professional work: practices, policies and organization. Int. J. Odontostomat., 14(4):581-585, 2020.

ABSTRACT: This article reflects on how current knowledge society understands the concept of "university governance", with regard to scientific production. Similarly, we propose that scientific work of the universities is dynamic and should present modifications according to the social changes and transformations of the times, which institutions of higher education also experience.

KEY WORDS: governance, management, scientific productivity.

\section{REFERENCIAS BIBLIOGRÁFICAS}

Alzate Zuluaga, M. L. \& Romo Morales, G. El enfoque de la gobernanza y su recepción en el marco gubernativo actual de las sociedades latinoamericanas. Opin. Pública, 20(3):480-95, 2014.

Arias Arbelaez, F. A. \& Vargas, G. M. Instituciones, gobernanza y sustentabilidad en la política colombiana de ordenamiento territorial municipal. Soc. Econ., (19):279-304, 2010. 
FUENTES, F. R.; DEL VALLE, R. C.; RIQUELME, M. C. \& CALLE, R. M. Reflexiones y propuestas en torno al dilema entre campo disciplinar y quehacer profesional: prácticas, políticas y organización. Int. J. Odontostomat., 14(4):581-585, 2020.

Blondeau, O.; Dyer, N.; Vercelone, C.; Corsani, A.; Rullani, E.; Boutang, \& Lazzarato, M. Capitalismo Cognitivo, Propiedad Intelectual y Creación Colectiva. Madrid, Traficantes de Sueños, 2014.

Bourdieu, P. Homo Academicus. Buenos Aires, Siglo XXI, 2003.

Brunner, J. J. \& Pedraja-Rejas, L. Challenges to higher education governance in Ibero-America. Ingeniare Rev. Chil. Ing., 25(1):24, 2017.

Castillo-Cubillos, M. El papel de la participación ciudadana en las políticas públicas, bajo el actual escenario de la gobernanza: reflexiones teóricas. C. S., 23:157-80, 2017.

De Sousa, B. Más Allá del Pensamiento Abismal: de las Líneas Globales a una Ecología de Saberes. En: Olivé, L.; de Sousa Santos, B.; de la Torre, C. S.; Antezana, L. H.; Navia Romero, W.; Tapia, L.; Valencia García, G.; Puchet Anyul, M.; Gil, M.; Aguiluz Ibargüen, M.; et al. (Coords.). Pluralismo Epistemológico. La Paz, CLACSO, 2019.

Fossatti, P. \& Danesi, L. C. Universidades comunitarias en Brasil: ¿por qué hay que perfeccionar su modelo de gestión?. Form. Univ., 11(5):75-84, 2018.

Ganga, F.; Quiroz, J. \& Fossatti, P. Análisis sincrónico de la gobernanza universitaria: una mirada teórica a los años sesenta y setenta. Educ. Pesqui., 43(2):553-68, 2017.

Horkheimer, M. \& Adorno, T. Dialéctica de la llustración. Fragmentos filosóficos. Madrid, Trotta, 1998.

Mollis, M. Geopolítica del Saber: Biografías Recientes de las Universidades Latinoamericanas. En: Vessuri, H. (Ed.). Universidad e Investigación Científica. Buenos Aires, CLACSO, 2006.

Olivé, L. Por una Auténtica Interculturalidad Basada en el Reconocimiento de la Pluralidad Epistemológica. En: Olivé, L.; de Sousa Santos, B.; de la Torre, C. S.; Antezana, L. H.; Navia Romero, W.; Tapia, L.; Valencia García, G.; Puchet Anyul, M.; Gil, M.; Aguiluz Ibargüen, M.; et al. (Coords.). Pluralismo Epistemológico. La Paz, CLACSO, 2019.

Rock, J. A. \& Rojas, C. Cambios en el sistema Universitario chileno: reflexiones sobre su evolución y una propuesta de gobernanza. Cal. Educ., (37):163-88, 2012.

Schmal, R. \& Cabrales, F. El desafío de la gobernanza universitaria: el caso chileno. Ensaio Aval. Pol. Publ. Educ., 26(100):82248, 2018.

Virgili Lillo, M.; Ganga Contreras, F. \& Figueroa Aillanir, K. Gobernanza universitaria o cogobierno: El caso de la Universidad de Concepción de Chile. Ultim. Decada, 23(42):187-216, 2015.
Dirección para correspodencia:

Prof. Dr. Ramón Fuentes Fernández

Research Centre for Dental Sciences

Dental School

Universidad de La Frontera

Av. Francisco Salazar 1145

Temuco

CHILE

Email: ramon.fuentes@ufrontera.cl

Recibido : 30-03-2020

Aceptado: 08-05-2020 